Historic, Archive Document Do not assume content reflects current
scientific knowledge, policies, or practices. 



\section{FARMERS' WHOLESALE PRICES APRIL 2, 1928}

ALFALFA

$1 / 2$ BU. $1 / 4$ BU.

Utility (Prime) Northwestern Grown........\$10.00 \$ $\$ 5.10 \$ 2.60$ Economy (Choice) Northwestern Grown.........111.00 $5.60 \quad 2.90$ Universal (Fancy) Northwestern Grown $3.12 .00 \quad 6.10 \quad 3.10$ Pinnacle (Extra Fancy) Northwestern Grown... 13.00 $\quad 6.60 \quad 3.40$ Dakota Grown No. 12 (Pinnacle).............

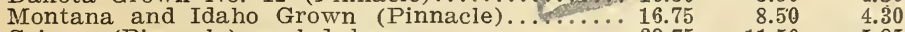

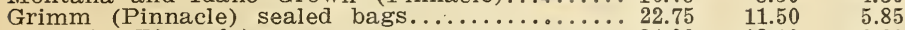

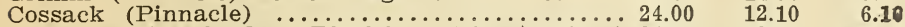
State Certified \& Idaho Blackfoot Grimm (Pinn.) 24.00 $12.10 \quad 6.10$ Ontario Variegated (Pinnacle)..............20.50 $10.35 \quad 5.25$

\section{MEDIUM RED CLOVER}

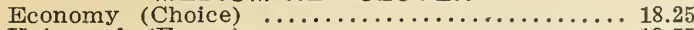

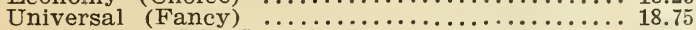

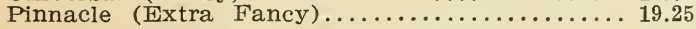

$9.15 \quad 4.6 \%$

$9.45 \quad 4.80$

MAMMOTH RED CLOVER

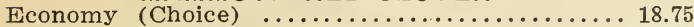

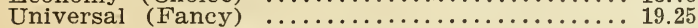

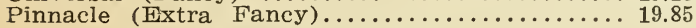

$9.65 \quad 4.95$

\section{ALSIKE CLOVER}

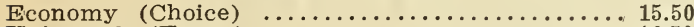

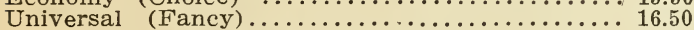

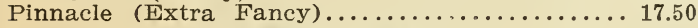

7.85

$\begin{array}{ll}8.85 & 4.25 \\ & 4.50\end{array}$

\section{SWEET CLOVER}

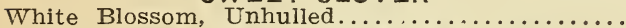

Utility, White Blossom, Hulled, 60 lb. bu..... 4.00

Economy White Blossom, Hulled, 60 ib. bu... 4.50

Universal White Blossom, Hulled, $60 \mathrm{lb}$. bu.... 4.80

Pinnacle Ex. Fancy White Blossom, 60 lb. bu... 5.40

Biennial Yellow Blossom (Pinnacle) $60 \mathrm{lb}$. bu.. 5.40

Grundy County Dwarf White (Pinn.) 60 ib. bu 5.40

Annual Yellow Blossom (Pinnacle) 60 lb. bu.. 4.00

\section{OTHER CLOVERS}

BU.

Hubam (Annual White Sweet Clover) 60 lb. bu. 16.50 Japan Clover, 25 lb. bu.................... 4.75

White Dutch Clover, 60 lb. bu.............25.00

Crimson or Giant Incarnate, 60 - ib bu.............. 12.00

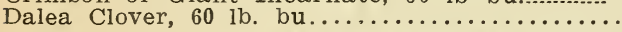

TIMOTHY, CLOVER \& TIMOTHY MIXTURES

Timothy, Utility, (Prime) $\ldots \ldots \ldots \ldots \ldots \ldots \ldots \ldots$

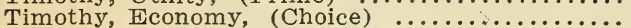

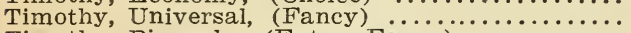

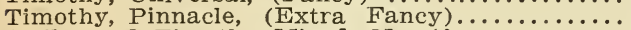

Alsike and Timothy Mixed, No. $10 \ldots \ldots \ldots \ldots \ldots$

Alsike and Timothy Mixed, No. $20 \ldots \ldots \ldots \ldots \ldots$.

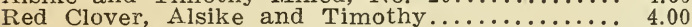

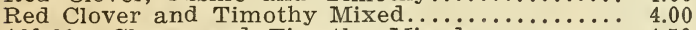

Alfalfa, Clover and Timothy Mixed.............. 4.50

GRASS MIXTURES AND FORAGE CROPS CWT.

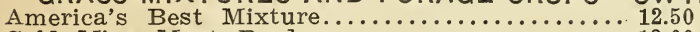

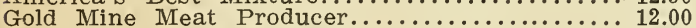

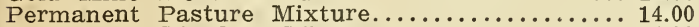

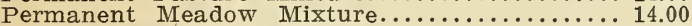
Annual Hog Pasture Mixture.............. 4.00

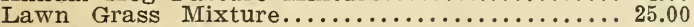

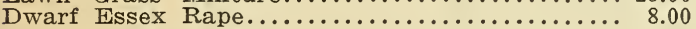

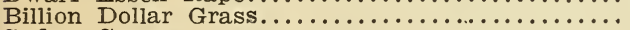

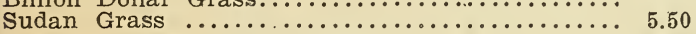

2.10

2.30

2.50

2.80

2.80

2.80

2.10

2 BU. 1/4 BU.

$8.35 \quad 4.25$

$2.45 \quad 1.35$

$12.60 \quad 6.40$

$6.20 \quad 3.15$

SOLD OUT

$\begin{array}{ll}1.10 & .60 \\ 1.20 & .70 \\ 1.30 & .75 \\ 1.40 & .80 \\ 1.90 & 1.00 \\ 2.40 & 1.25 \\ 2.10 & 1.10 \\ 2.13 & 1.10 \\ 2.35 & 1.25\end{array}$

50 LB. 25 LB.

$6.35 \quad 3.25$

$6.10 \quad 3.10$

$7.10 \quad 3.65$

$7.10 \quad 3.65$

$2.10 \quad 1.10$

$12.75 \quad 6.50$

$4.10 \quad 2.10$

$2.85 \quad 1.50$

$10.15 \quad 5.20$

$10.80 \quad 5.50$

$8.35 \quad 4.25$

$10.60 \quad 5.40$

$6.60 \quad 3.40$

$10.10 \quad 5.10$

$7.10 \quad 3.65$

$8.10 \quad 4.10$

$6.35 \quad 3.50$

$7.10 \quad 3.65$

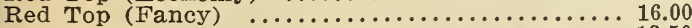

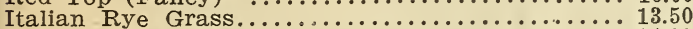

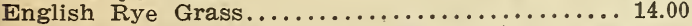




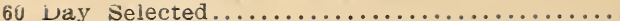

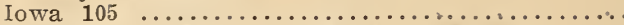

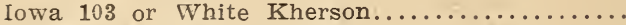

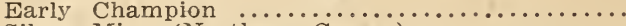

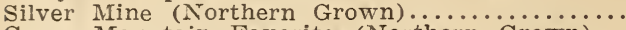
Green Mountain Favorite (Northern Grown)... Regenerated Swedish Select (Canadian Grown).

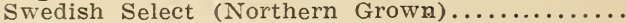

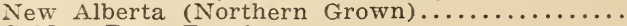

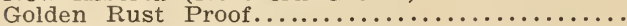

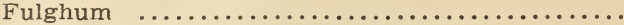

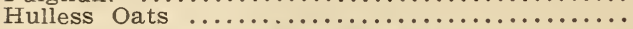

\section{OTHER SEED GRAINS}

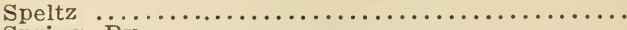
Spring Rye $\ldots \ldots \ldots \ldots \ldots \ldots \ldots \ldots \ldots \ldots \ldots \ldots$

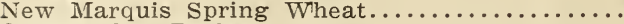

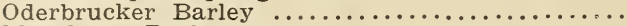

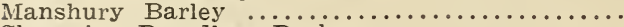

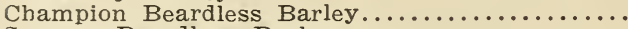

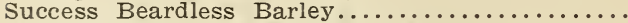

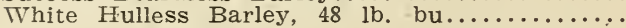

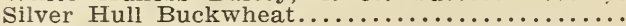
Japanese Buckwheat

\section{FORAGE CROPS}

Cane (Orange)

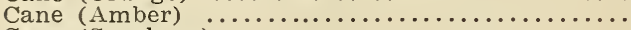

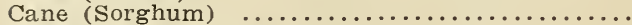

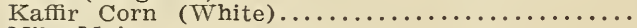

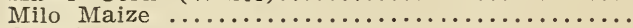

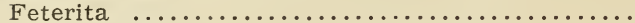

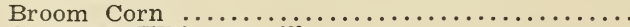

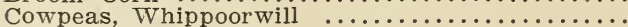

Cowpeas, New Era $\ldots \ldots \ldots \ldots \ldots \ldots \ldots \ldots \ldots \ldots$

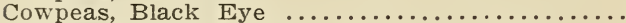

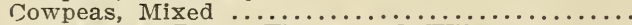

Soy Beans, Black Ebony and Wilson...........

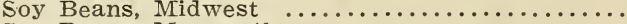

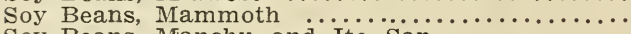

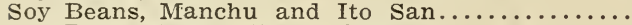

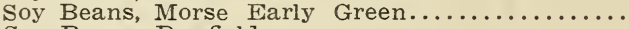

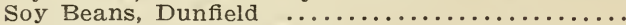

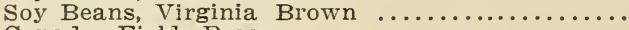

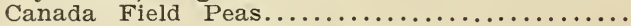

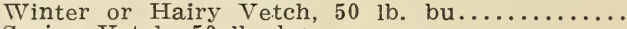

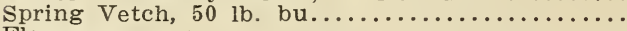

Flax

MILLETS

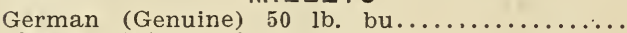

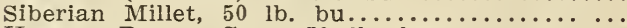

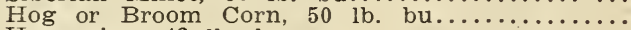

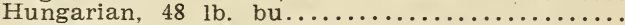

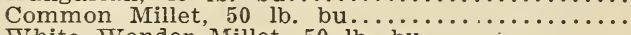

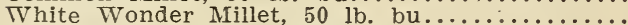

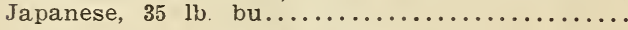

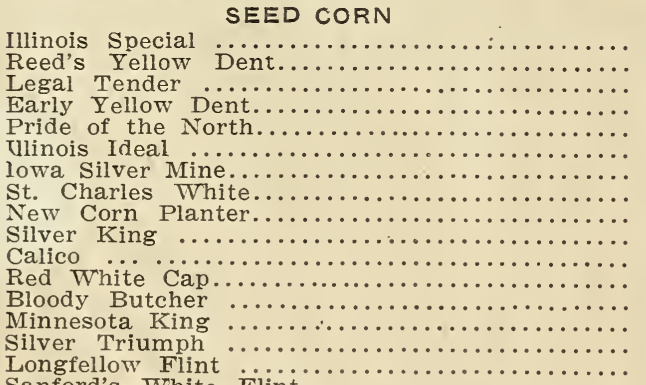

\begin{tabular}{rrr}
1 BU. & \multicolumn{1}{c}{ B $B$. $1 / 4$ BU. } \\
3.75 & 1.95 & 1.00 \\
3.50 & 1.85 & .95 \\
3.50 & 1.85 & .95 \\
3.50 & 1.85 & .95 \\
3.50 & 1.85 & .95 \\
3.75 & 1.95 & 1.00 \\
3.50 & 1.85 & .95 \\
3.50 & 1.85 & .95 \\
3.75 & 1.95 & 1.00 \\
3.50 & 1.85 & .95 \\
3.50 & 1.85 & .95 \\
3.50 & 1.85 & .95 \\
3.50 & 1.85 & .95 \\
3.50 & 1.85 & .95 \\
3.65 & 1.90 & 1.00 \\
3.65 & 1.90 & 1.00 \\
3.65 & 1.90 & 1.00
\end{tabular}

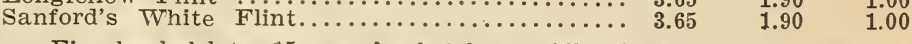

Five bushel lots, 15c per bushel less. All prices include bags free.

Cyclone seeders, $\$ 2.00$ each; $\$ 2.25$ postpaid.

\section{AMERICAN FIELD SEED CO.

